\title{
Neuartige Maschinenelemente in der Fördertechnik und Logistik : Hochfeste, laufende Faserseile
}

\author{
DR.-ING. WOLFRAM VOGEL, \\ PROF. DR.-ING. KARL-HEINZ WEHKING \\ UNIVERSITÄT STUTTGART, INSTITUT FÜR FÖRDERTECHNIK UND LOGISTIK (IFT)
}

Materialflusssysteme und fördertechnische Anlagen und Maschinen lassen sich herunterbrechen auf die Fördermittel, die Groß- und Unterbaugruppen bis hin zu den Basiselementen wie z.B. Tragmittel, Bremse, Kupplung, Zahnrad und Antrieb (Abb. 1). Eine sehr wesentliche sicherheitstechnische Funktion kommt dem Tragmittel zu. Das am häufigsten in verschiedensten Anwendungsfällen wie Kran, Aufzug, Seilbahn, Hebezeugen usw. eingesetzte Tragmittel ist bisher noch mit Abstand das Stahldrahtseil. Dabei wird zwischen vorwiegend auf zug- und zugschwellbeanspruchte Seile und schwellend auf Biegung beanspruchte über Scheiben laufende Seile unterschieden. Im Bereich der modernen Wachstumsbranchen Fördertechnik und Logistik steigen die Anforderungen an die Tragmittel Seile hinsichtlich der Lebensdauer, der Ablegereifeerkennung, der Reduzierung der Masse bei gleichzeitiger Steigerung von Bruchkräften, Verzicht auf Schmierung bis hin zu gekoppelten Funktionalitäten aus Kraft übertragen, Beanspruchung messen, Daten übertragen und Selbstüberwachung des Seils. Die hohen Anforderungen an die nächsten Generationen Tragmittel sind nicht ausschließlich mit Stahldrahtseilen zu erfüllen. Vielmehr müssen neuartige Maschinenelemente aus spezifischen Materialien mit angepassten Eigenschaften als Tragmittel in der Materialflusstechnik und Logistik Einzug halten.

Material flow systems, conveyors and machines consist of the conveyor, main and subcomponents and basic elements such as load-carrier, break, clutch, gearwheel and drive. With regard to security the load-carrier plays a major role. The load-carrier which is most often used in cranes, lifts, cablecars, hoisting gears etc. is the steel wire rope. Here, it is differentiated between ropes which are submitted to tensile and pulsating tensile stresses and ropes which are submitted to pulsating bending stress and run over washers. The modern branch of material handling and logistics puts high requirements on the load-carriers with regard to their longevity, determination of the replacement state of wear, mass reduction in line with improved breaking resistance, abandonment of lubrication up to a coupling of the functions transmission of forces, measuring of stress, data transfer of self-control. These high requirements could not be satisfied by steel wire ropes alone. New machine elements of specific materials and with specific features have to be developed as load-carriers for material flow and logistics.

\section{1.}

Hierzu ist es notwendig, gezielte Neuentwicklungen durchzuführen. Fingerzeige sind durch den Einsatz von hochfesten, laufenden Faserseilen in verschiedenen sicherheitsrelevanten Anwendungen bereits gegeben. Über die wenigen dazu bisher durchgeführten wissenschaftlichen Untersuchungen zu laufenden Faserseilen in sicherheitsrelevanten Anwendungsbereichen wird im Folgenden berichtet. Zudem werden die Parallelen, aber auch die Defizite der hochfesten Fasereile zu laufenden Stahldrahtseilen aufgezeigt, die im Wesentlichen aus fehlenden experimentellen Reihenuntersuchungen resultieren und einen breiten Einsatz von hochfesten, laufenden Faserseilen in sicherheitsrelevanten Anwendungen aber auch komplexen Materialflusssystemen derzeit praktisch noch verhindern. Um solche verbesserten Maschinenelemente „Faserseile“ in der Breite der Anwendungen einsetzen zu können, sind Untersuchungen notwendig, zu denen Zug- und Zugschwellversuche mit verschiedenen Endverbindungen, Dauerbiegeversuche, Treibfähigkeitsversuche aber auch weitergehende Versuche mit besonderen mechanischen Beanspruchungen und unterschiedlichen Umgebungsbedingungen unbedingt notwendig. Intensive Zusammenarbeiten zwischen Herstellern, Anwendern und Forschungsstellen sind dazu unerlässlich. 
FÖRDERMITTEL:

GROBBAUGRUPPEN:

KLEINBAUGRUPPEN:

BAUELEMENTE:
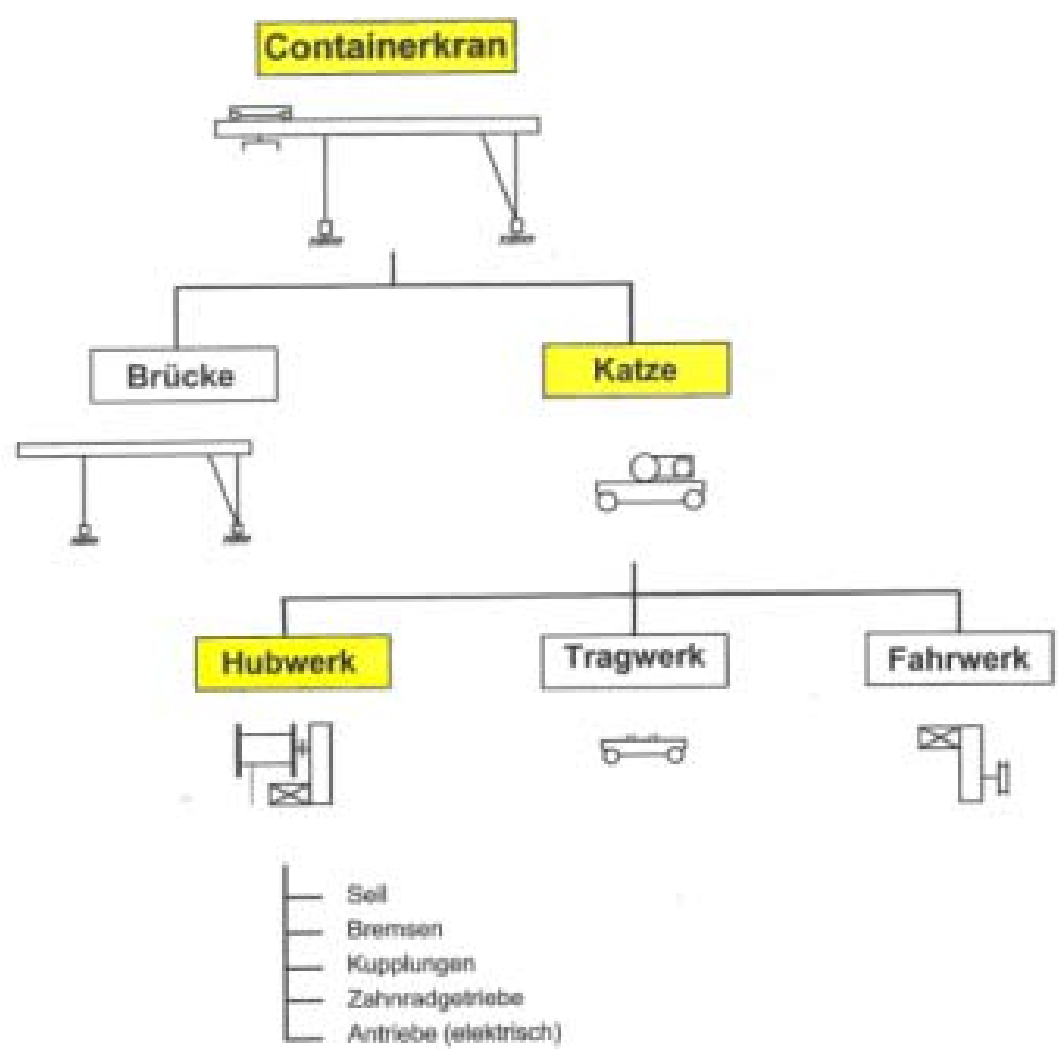

Abbildung 1: Vom Fördermittel zum Bauelement

\section{Anforderungen an laufende Faserseile}

Bei den laufenden Seilen müssen die sicherheitsrelevanten Anforderungen

- ausreichende Lebensdauer

- zuverlässige Erkennung der Seilablegereife, d.h. Erkennung des Zeitpunkts für den Seiltausch, und zwar rechtzeitig, bevor ein gefährlicher Zustand eintritt

- bei Treibscheibenantrieben ausreichende Treibfähigkeit

erfüllt werden. Dies gilt in gleichem Maße auch für laufende Faserseile. Weitere Anforderungen, wie die Federund Dämpfungseigenschaften der Seile, das Dehnverhalten und die Laufruhe sind hinsichtlich der sicherheitstechnischen Anforderungen untergeordnet, bestimmen aber wesentlich die Gebrauchseigenschaften, den Komfort und die Akzeptanz der Produkte am Markt.

\section{Lebensdauer und Ablegereife laufender Faserseile}

Die Welt der Stahldrahtseile kennt für die laufenden Seile praktisch nur Drähte aus unlegierten Kohlenstoffstählen und in Ausnahmen aus Edelstählen. Die Zahl der Konstruktionen bei Stahldrahtseilen ist vielfältig aber noch überschaubar. Bei den Faserseilen hingegen sind weit mehr Konstruktionen bekannt. Vor allem sind aber viele Faserwerkstoffe im Einsatz, deren Gebrauchseigenschaften z.B. durch Fertigungsprozesse und / oder Nachbehandlungen der Fasern und auch des kompletten Seils in sehr weiten Grenzen einstellbar sind. Die über Scheiben laufenden Seile werden durch ein Beanspruchungskollektiv, d.h. schwellende Biege- und Zugspannungen, aber auch Pressung und Ovalisierungsspannungen beansprucht.

Die Seillebensdauer beim Lauf über Scheiben ist von den Seilparametern wie

- Seilkonstruktion

- Seildurchmesser d

- Seileinlage

- Draht- und Faserfestigkeit

- Schmierung, etc.

und zahlreichen Anlagenparametern wie

- Seilzugkraft S

- Verhältnis Scheibendurchmesser zu Seilnenndurchmesser D/d 
- Biegelänge 1

- Ablenkwinkel

- Rillenform

- Schrägzugwinkel

- Rillenwerkstoff

- Art der Biegung

- Mehrlagenwicklung

- Kombination von Zug- und Biegebeanspruchungen, etc.

abhängig.

Der Lauf des Seiles ist nur deshalb leicht möglich, weil die Seildrähte und die tragenden Fasern gegeneinander verschiebbar sind. Durch diese Relativbewegung tritt aber an den Drähten und den Fasern Verschleiß in verschiedenen Erscheinungsformen auf. Aufgrund des Drahtverschleißes kann selbst bei genauer Kenntnis aller Draht- und Faserspannungen damit die Seillebensdauer nicht berechnet werden.

Die sicherheitsrelevanten Größen Lebensdauer und Ablegereife, d.h. die Biegewechselzahlen von Stahldrahtseilen bis zum Bruch der Seile N bzw. bis zum Austausch der Seile rechtzeitig vor dem Seilbruch $\mathrm{N}_{\mathrm{A}}$, können vielmehr nur in Dauerbiegeversuchen ermittelt werden.

\section{Dauerbiegeversuche - Seile auf „Seele und Litze“ geprüft}

Der Vergleich in der Überschrift hinkt in keiner Weise. Was beim Menschen für „Herz und Niere“ steht, sind beim Seil die wichtigen Bauteile „Seele und Litze“. Für die Dauerbiegeversuche an Seilen werden Dauerbiegemaschinen eingesetzt, die schematisch in Abbildung 2 dargestellt sind. Das Seil ist in der Dauerbiegemaschine um die Prüf- und die Seiltreibscheibe geschlungen. Durch die oszillierende Hin- und Herbewegung der Seiltreibscheibe läuft das Seil auf der Prüfscheibe auf und ab, nimmt dabei die Zustände gerade - gekrümmt - gerade ein und wird damit durch Biegewechsel beansprucht. Während der Dauerbiegeversuche wird die Prüfscheibe über einen Hebel und starre Massen belastet, so dass im Seil eine konstante Zugkraft wirkt. Die Biegefrequenz bei den Dauerbiegeversuchen wird so eingestellt, dass sich die Versuchsseile auf nicht mehr als $50^{\circ} \mathrm{C}$ erwärmen. Im Labor des Instituts für Fördertechnik und Logistik der Universität Stuttgart (IFT) werden die Dauerbiegeversuche üblicherweise bei Raumtemperatur, trockener Umgebung, Prüfscheiben mit Rundrillen, einem Rillenradius $r=0,53$ x Seilnenndurchmesser $d$ und einem Rillenöffnungswinkel von $60^{\circ}$ durchgeführt, bis das Seil oder mindestens eine Litze gebrochen ist. Der Einfluss von davon abweichenden Bedingungen wird durch Korrekturfaktoren berücksichtigt, die aus speziellen Laborversuchen und Feldbeobachtungen abgeleitet wurden.

Während der Dauerbiegeversuche werden die Versuche bei Biegewechseln entsprechend der Normzahlreihe R10 angehalten, um den Seilzustand in regelmäßigen Abständen zu inspizieren. Bei diesen regelmäßigen Inspektionen werden Drahtbrüche auf Bezugslängen ermittelt, Durchmesser gemessen und Seilveränderungen festgehalten, die zur Ermittlung der Ablegereife und Ablegereifekriterien herangezogen werden. 


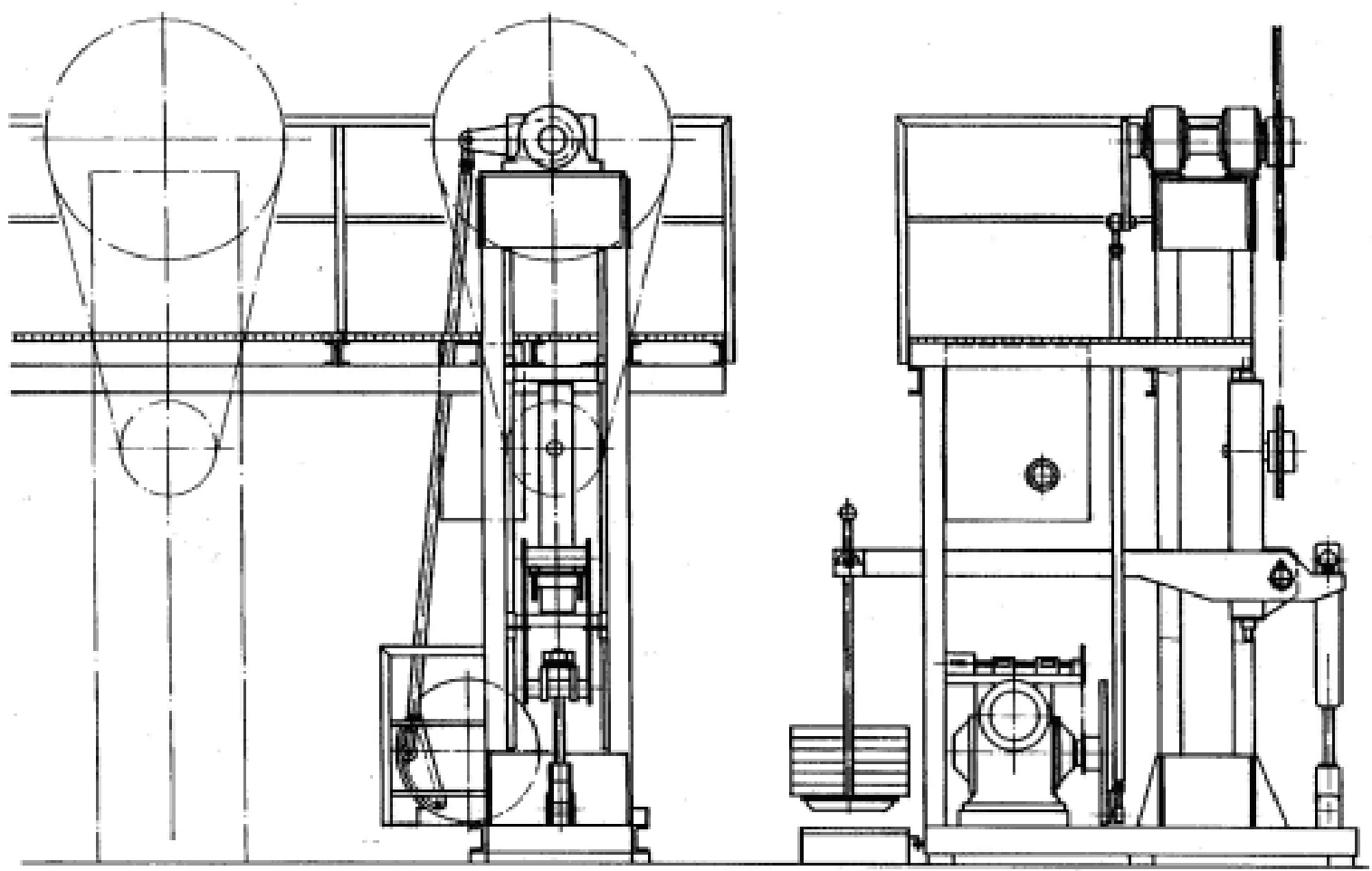

Abbildung 2: Skizze einer IFT-Dauerbiegemaschine mit fliegender Prüfscheibe

Tabelle 1: Untersuchte Eigenschaften von laufenden Seilen

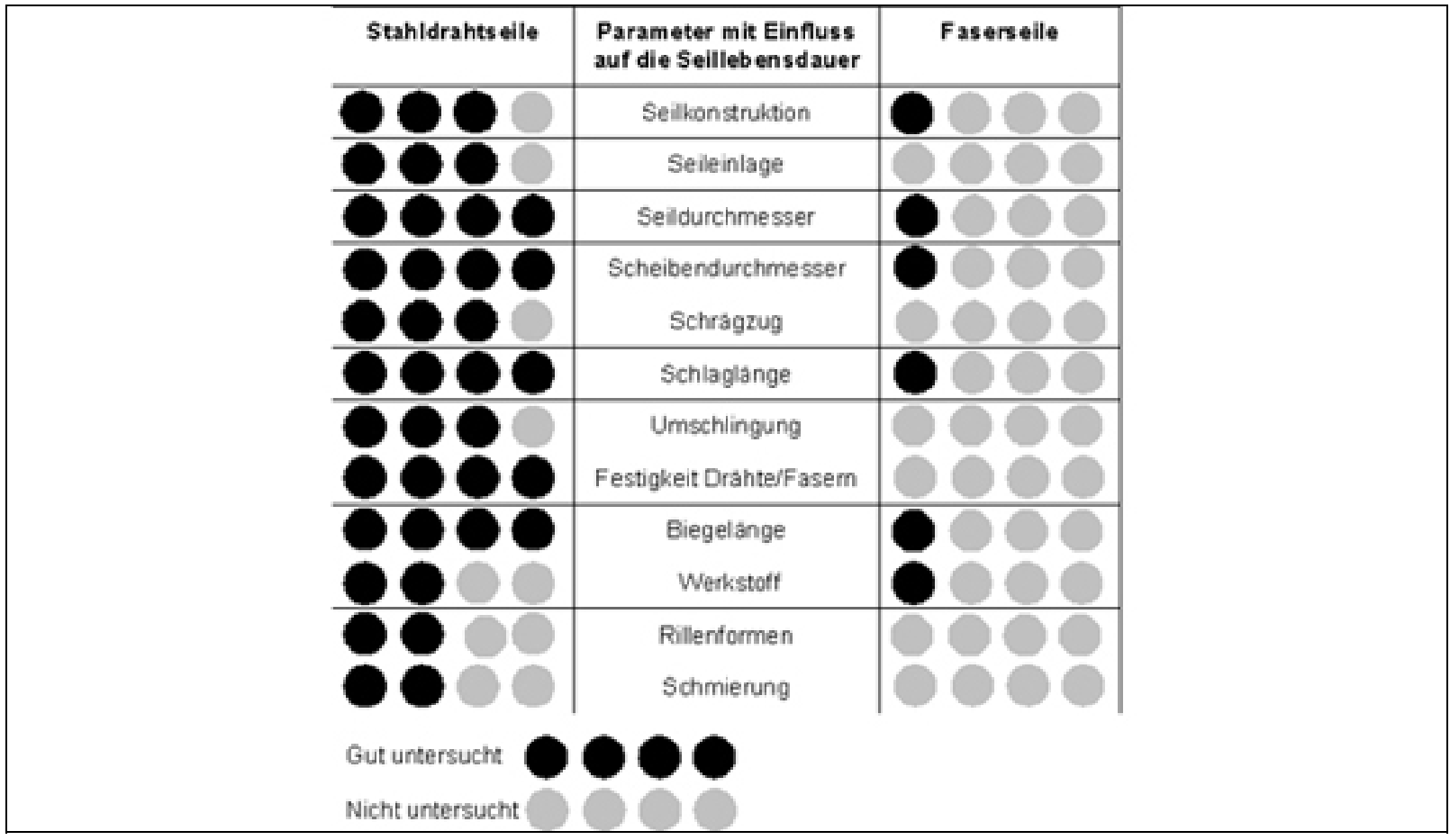

Die Lebensdauer von Stahldrahtseilen kann für die häufig eingesetzten und die genormten Stahldrahtseile durch die Lebensdauergleichung und -berechnungsmethode für Stahldrahtseile nach [Feyrer00] in nahezu beliebigen Seiltrieben mit sehr guter Näherung berechnet werden.

Für laufende Faserseile fehlen diese Möglichkeiten der Berechnung der Seillebensdauer in der Breite der sicherheitsrelevanten Anwendungen praktisch vollständig, von wenigen punktuellen Ausnahmen [Vogel91Vogel03; Wehking98; Wehking00] abgesehen. Tabelle 1 zeigt deutlich die Defizite bei der systematischen Untersuchung laufender Faserseile im Vergleich zu den noch üblicherweise eingesetzten Stahldrahtseilen. 


\section{Beispiele für Untersuchungen an laufenden Faserseilen}

\section{Bruchbiegewechselzahlen}

Die Untersuchungen laufender Faserseile am IFT sind im wesentlichen durch sehr spezielle Fragestellungen der Industrie mittels Drittmittelaufträgen aus den Bereichen Serienhebezeuge, Offshore-Technik, Aufzugbau etc. zurückzuführen.

Im Bereich der Serienkleinhebezeuge ist ein Lastenheft für den Einsatz eines Faserseiles in einem Treibantrieb erstellt und eine Gefahrenanalyse durchgeführt worden. Als wesentliche Gefährdungsereignisse sind bei dieser Gefahrenanalyse das Scheuern des Faserseiles an Bauteilen des Hebezeuges und scharfen Kanten der Arbeitsumgebung erkannt worden. Für den Treibantrieb bestehend aus der Paarung Faserseil - Kunststoffscheibe war unklar, ob bei allen denkbaren aber relevanten Umgebungsbedingungen die Treibfähigkeit gegeben ist und ein Lastabsturz infolge eines durchziehenden Seiles ausgeschlossen werden kann. Da ein nasses oder zumindest feuchtes Seil z.B. durch Arbeiten bei geöffneten Toren, Kondensation von Wassernebel etc. denkbar ist, wurden Hebevorgänge mit - den Extremfall betrachtend - vollständig gewässerten Seilen durchgeführt. Es konnte festgestellt werden, dass ein Heben der Last mit gewässertem Seil nicht möglich ist und - sicherheitstechnisch sehr bedeutend - die zu hebende Masse auch bei abgeschaltetem Antrieb in der Schwebe gehalten wird. Bei laufendem Antrieb dreht die Treibscheibe unter dem Seil durch. Auch nach längerem, weit über der Praxis liegendem Durchdrehen der Scheibe konnte in anschließenden Zugversuchen praktisch kein Bruchkraftverlust des Faserseils festgestellt werden. Das untersuchte Serienkleinhebezeug HandyLifter von DEMAG ist nach entsprechenden Messeauftritten, z.B. der Industriemesse Hannover, am Markt erfolgreich etabliert worden.

Im Offshore-Bereich werden für verschiedene Anwendungen, wie dem Verlegen von Unterwasserkabeln etc., leichte und schwimmfähige - damit den Antrieb verkleinernde - Seile mit hohen Bruchkräften gefordert. Da die Seile im Betrieb über Scheiben laufen und aufgetrommelt werden, sind zudem gute Biegewechseleigenschaften gefordert. Am IFT ist im Dauerbiegeversuch (Abb. 4) das in Abbildung 3 gezeigte Faserseil geprüft worden.

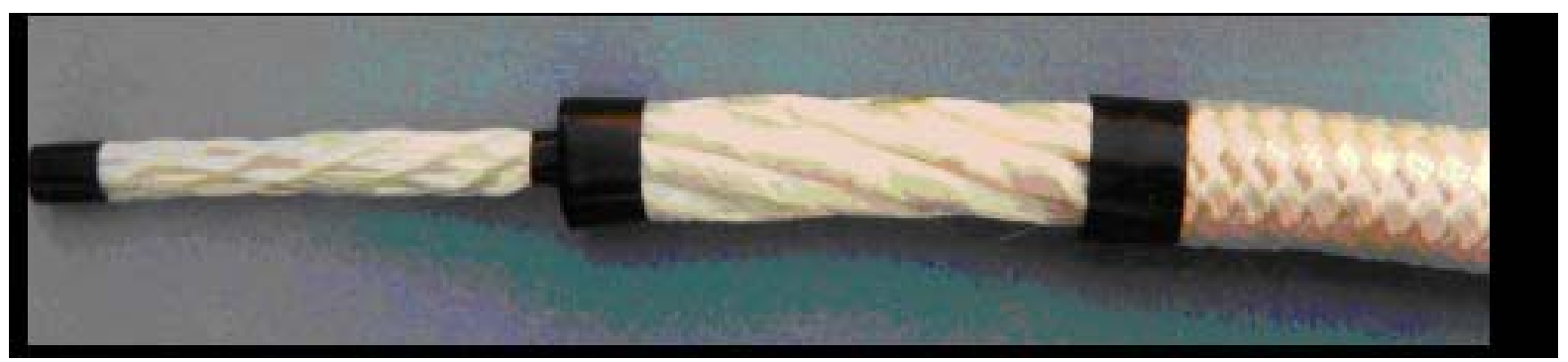

Abbildung 3: Faserseil mit tragenden Litzen aus hochfesten Polyethylenfasern Dyneema SK60

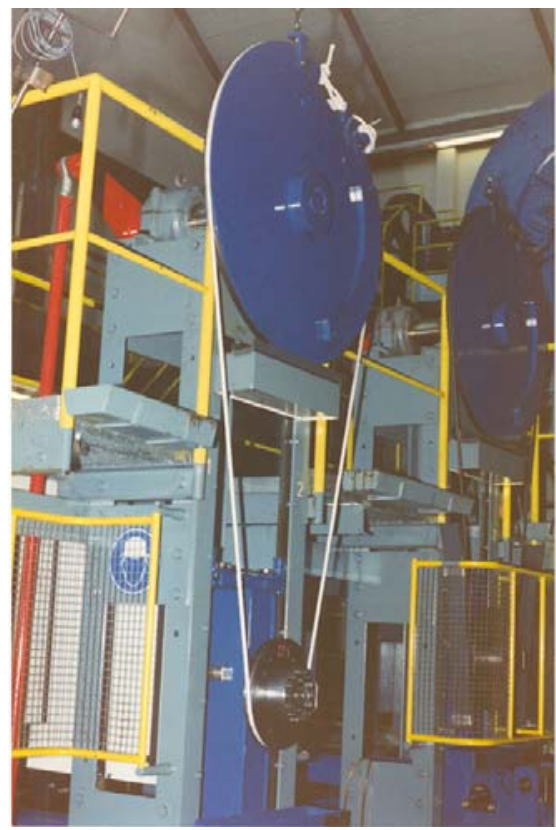




\section{Abbildung 4: Biegeversuch mit Faserseil auf einer Stahlscheibe}

In Abbildung 5 sind die in Dauerbiegeversuchen ermittelten Bruchbiegewechselzahlen $\mathrm{N}$ über der durchmesserbezogenen Seilzugkraft $\mathrm{S} / \mathrm{d}^{2}$ für die untersuchten Durchmesserverhältnisse D/d doppellogarithmisch aufgetragen.

Mit zunehmender Seilzugkraft und konstantem Durchmesserverhältnis von Scheibe zu Seil D/d nimmt die Bruchbiegewechselzahl ab. Für eine konstante Seilzugkraft nimmt die Bruchbiegewechselzahl mit größer werdendem D/d, d.h. kleiner werdender Biegespannung in den Filamenten, stark zu. Die Bruchbiegewechselzahlen lassen sich für kleine und mittlere durchmesserbezogene Seilzugkräfte durch Geraden mit mäßiger Steigung beschreiben:

$$
\lg \bar{N}=b_{0}+b_{1} \lg \frac{S d_{0}^{2}}{d^{2} S_{0}}+b_{2} \lg \frac{D}{d}+b_{3} \lg \frac{S d_{0}^{2}}{d^{2} S_{0}} \lg \frac{D}{d}
$$

Die Regressionskoeffizienten $\mathrm{a}_{1}$ bis $\mathrm{a}_{4}$ sind mit der Methode der linearen Mehrfachregression speziell für das untersuchte Faserseil abgeleitet worden.

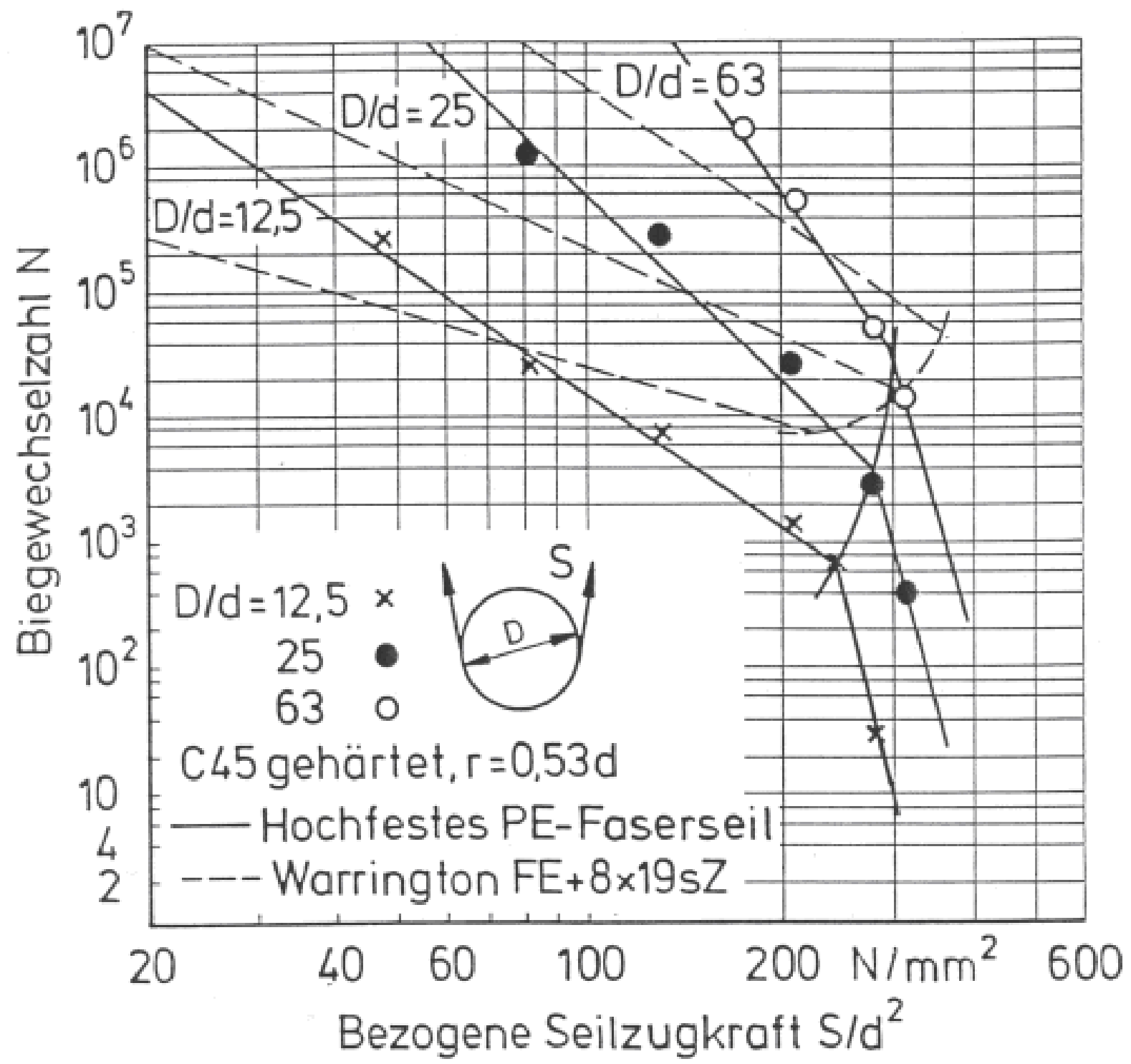


Abbildung 5: Bruchbiegewechselzahlen eines Faserseils mit hochfesten Polyethylenfasern „Dyneema SK60“ [Vogel02]

$\mathrm{Ab}$ der sogenannten Sprungspannung fallen die Bruchbiegewechselzahlen mit weiter steigender Seilzugkraft schroff ab. Der Dauerbruch geht in einen Gewaltbruch über. In Abbildung 5 sind zusätzlich die Bruchbiegewechselzahlen eines Stahldrahtseiles mit bekannt guten Biegewechseleigenschaften eingezeichnet. Es kann festgestellt werden, dass für kleine Seilzugkräfte die Faserseile den Stahldrahtseilen überlegen sind. Schon hier lässt sich Relevanz und Chance für laufende Faserseile in Seiltrieben erkennen.

$\mathrm{Zu}$ den wenigen im Dauerbiegeversuch untersuchten Seilparametern, außer der Seilzugkraft S und des Durchmesserverhältnisses D/d, zählt die Schlaglänge der im wesentlichen tragenden Litzen des in Abbildung 3 gezeigten Seils. Mit kleiner werdender Schlaglänge der Litzen und damit zunehmender Gleichmäßigkeit der Aufliegeverhältnisse des Seils in der Rille nehmen die Biegewechselzahlen zu (Abb. 6) [Vogel02]. Die Reduzierung der Schlaglänge muss aber ein Kompromiss sein zwischen guten Biegewechseleigenschaften und gewünschten Festigkeits- und Dehnungseigenschaften des Seils. In dieser Untersuchung ist bei einem Durchmesserverhältnis von Scheibe zu Seil D/d=25 die Schlaglänge l =85 mm, d.h. jede Litze liegt 5 mal in der Rille auf, gewählt worden.



Abbildung 6: Einfluss der Schlaglänge auf die Seillebensdauer bei dem hochfesten Faserseil aus Polyethylenfasern [Vogel02]

\section{Tiefseeforschung}

Bei der Untersuchung der Biegewechseleigenschaften hochfester Polyethylenfaserseile sind die Durchmesserverhältnisse $\mathrm{D} / \mathrm{d}$ und die Seilzugkräfte in weiten Grenzen variiert worden. Für eine Sonderanwendung in der Tiefseeforschung und dem sogenannten „deep sea mining“ laufen die Seile unter hohen Zugkräften über sehr kleine Scheiben. Um einen Verlust der teuren Ausrüstung, die im Tiefseeeinsatz gehoben und gesenkt werden soll, auszuschließen, sind mit geflochtenen und gedrehten Faserseilen aus hochfesten Polyethylenfasern Dauerbiegeversuche durchgeführt worden für ein konstantes Durchmesserverhältnis D/d, [Vogel98].

Die Biegewechselzahlen zeigen den bereits bekannten Verlauf über der Seilzugkraft, der für die geflochtenen und gedrehten Seile praktisch gleich ist (Abb. 7). Dieser so nicht ohne weiteres zu erwartende Effekt ist begründet in den hohen Biegespannungen infolge der kleinen Scheiben und der kleinen Reibung zwischen den Fasern. 

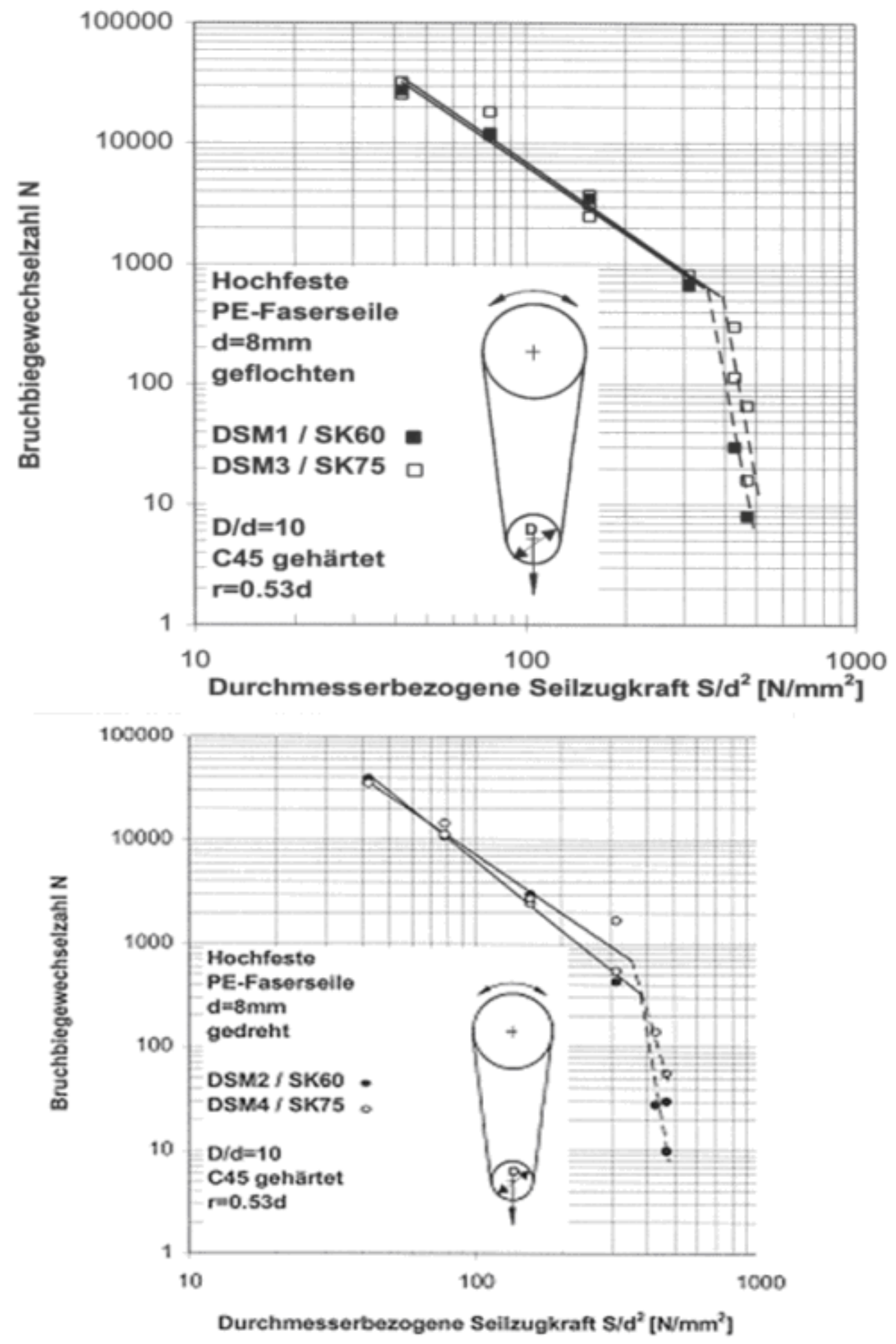

Abbildung 7: Bruchbiegewechselzahlen von gedrehten und geflochtenen Faserseilen bei kleinen Biegeverhältnissen [Vogel98]

\section{Schleppen und Festmachen von Schiffen}

Atlastaue, deren Konstruktion mit Litzen und Monofildrähten am ehesten an Drahtseilkonstruktionen erinnern, werden z.B. beim Schleppen und Festmachen von Schiffen, beim Festmachen einer Zug- und Zugschwellbeanspruchung aber auch einer Biegebeanspruchung ausgesetzt. Die Biegewechseleigenschaften von Atlastauen (aber nur sehr speziell mit Seilnenndurchmessern $d=50 \mathrm{~mm}$ ) sind am IFT in wenigen Dauerbiegeversuchen untersucht worden. Auch bei den Atlastauen hat sich die bereits gefundene lineare Abhängigkeit der Bruchbiegewechselzahl von der Seilzugkraft gezeigt. Abbildung 8 zeigt das Ende eines Dauerbiegeversuchs nach dem praktisch vollständigen Bruch des Seiles. 


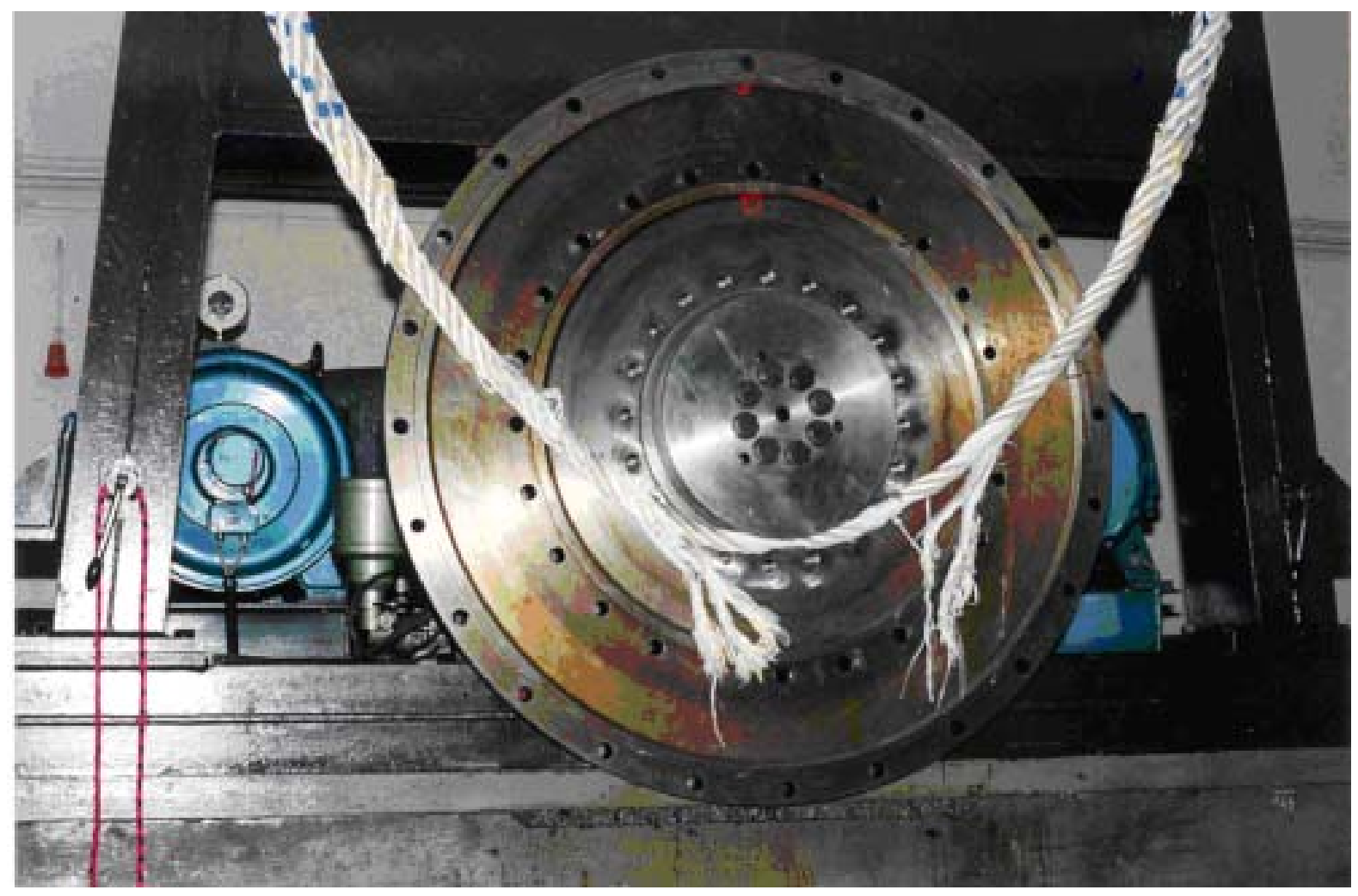

Abbildung 8: Dauerbiegeversuch mit Atlastau (Seilnenndurchmesser d=50mm) auf der Dauerbiegemaschine 5 des IFT (maximale Seilzugkraft $\mathrm{S}=500 \mathrm{kN}$ ) [Vogel02]

\section{Aufzugbau}

Für Treibscheibenaufzüge sind Aramidfaserseile der Schindler AG für die Anwendung im Treibscheibenaufzug zugelassen (Abb. 9). Mit den Aramidfaserseilen sind zahlreiche Dauerbiegeversuche auf den Dauerbiegemaschinen des IFT bei verschiedenen Seilzugkräften auf Prüfscheiben mit Rundrillen mit einem Durchmesserverhältnis bis herunter auf etwa $\mathrm{D} / \mathrm{d}=21$ durchgeführt worden [Wehking98], [Vogel03], [Wehking00]. Bei diesen Dauerbiegeversuchen sind Bruchbiegewechselzahlen teilweise bis zum 30-fachen der Bruchbiegewechselzahl eines Stahldrahtseiles mit guten Biegewechseleigenschaften bei sonst gleichen Versuchsbedingungen erreicht worden (Abb. 10). 


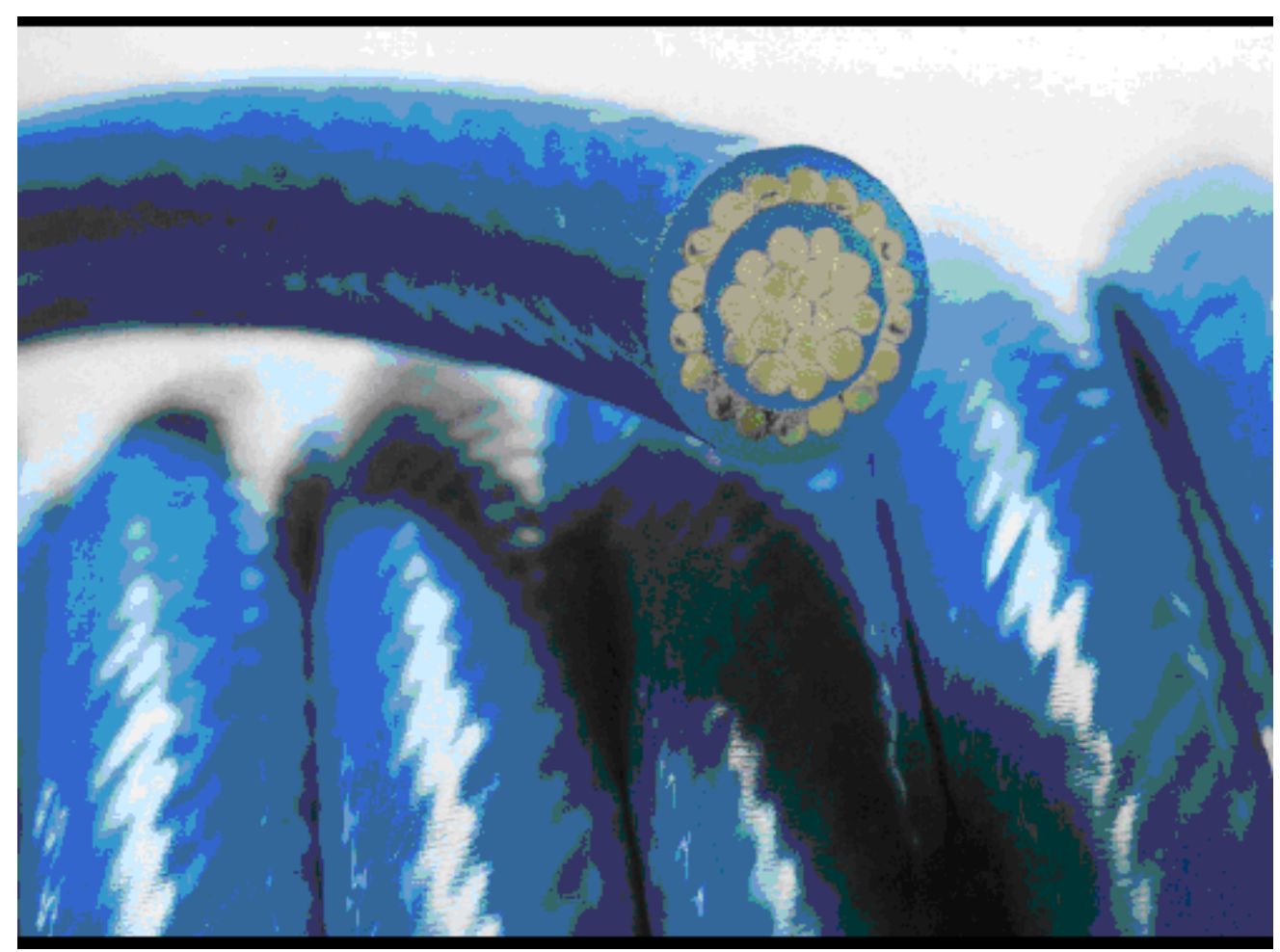

Abbildung 9: Aramidfaserseil für Treibscheibenaufzüge der Schindler AG

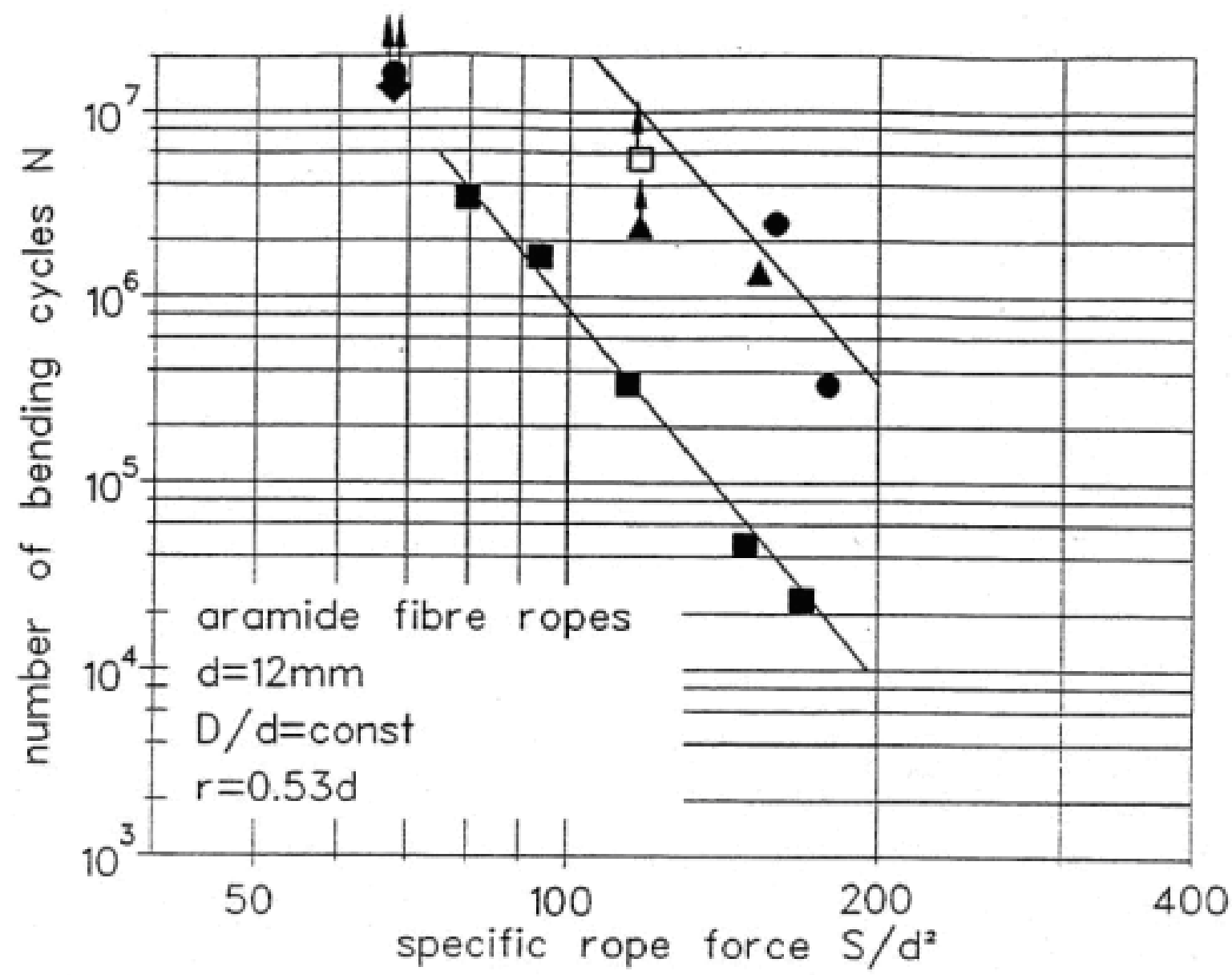




\section{Ablegereife}

Die Ablegereife der Seile, d.h. der rechtzeitige Tausch der Seile bevor ein gefährlicher Zustand für Mensch und Maschine entsteht, ist eine wesentliche sicherheitsrelevante Anforderung. Typische Ablegekriterien bei Faserseilen sind beispielhaft Garnbrüche, Litzenbrüche, Quetschungen, Schäden durch Schnitte, aggressive Medien, Durchmesseränderungen etc. Einige dieser Ablegekriterien wie die Seildurchmesseränderung wachsen mit der Zeit.

Für die in untersuchten hochfesten Polyethylenfaserseile sind bei den regelmäßigen Inspektionen während der Dauerbiegeversuche die Seildurchmesser in den Biegezonen gemessen und dokumentiert worden. In Abbildung 11 ist der relative Seildurchmesser $\mathrm{d}_{\mathrm{N}} / \mathrm{d}_{0}\left(\mathrm{~d}_{0}\right.$ ist der Seildurchmesser vor dem Versuch aber unter Zugbelastung) über der Biegewechselzahl $\mathrm{N}$ aufgetragen. Der relative Seildurchmesser nimmt mit zunehmender Biegewechselzahl zunächst nur mäßig und kurz vor dem Seilbruch sehr stark ab. Soll die Durchmesserabnahme als Ablegekriterium gelten, muss sie rechtzeitig vor dem Seilbruch erkennbar sein, wobei als angemessene Grenze 80\% der Lebensdauer angesehen werden kann. In Abbildung 11 ist zu erkennen, dass die Durchmesserabnahme von $10 \%$ bei $80 \%$ der Seillebensdauer nur bei kleineren Seilzugkräften und entsprechend hohen Lebensdauern erreicht wird.

Für den vorliegenden Fall war die Zuverlässigkeit der Ablegereifeerkennung mit einer entsprechenden statistischen Abgrenzung der Ablegebiegewechselzahlen gerade noch ausreichend. Ziel muss es aber sein, für die breite sicherheitsrelevante Anwendung von laufenden Faserseilen in Seiltrieben schärfere Ablegekriterien und grenzen zu definieren. 


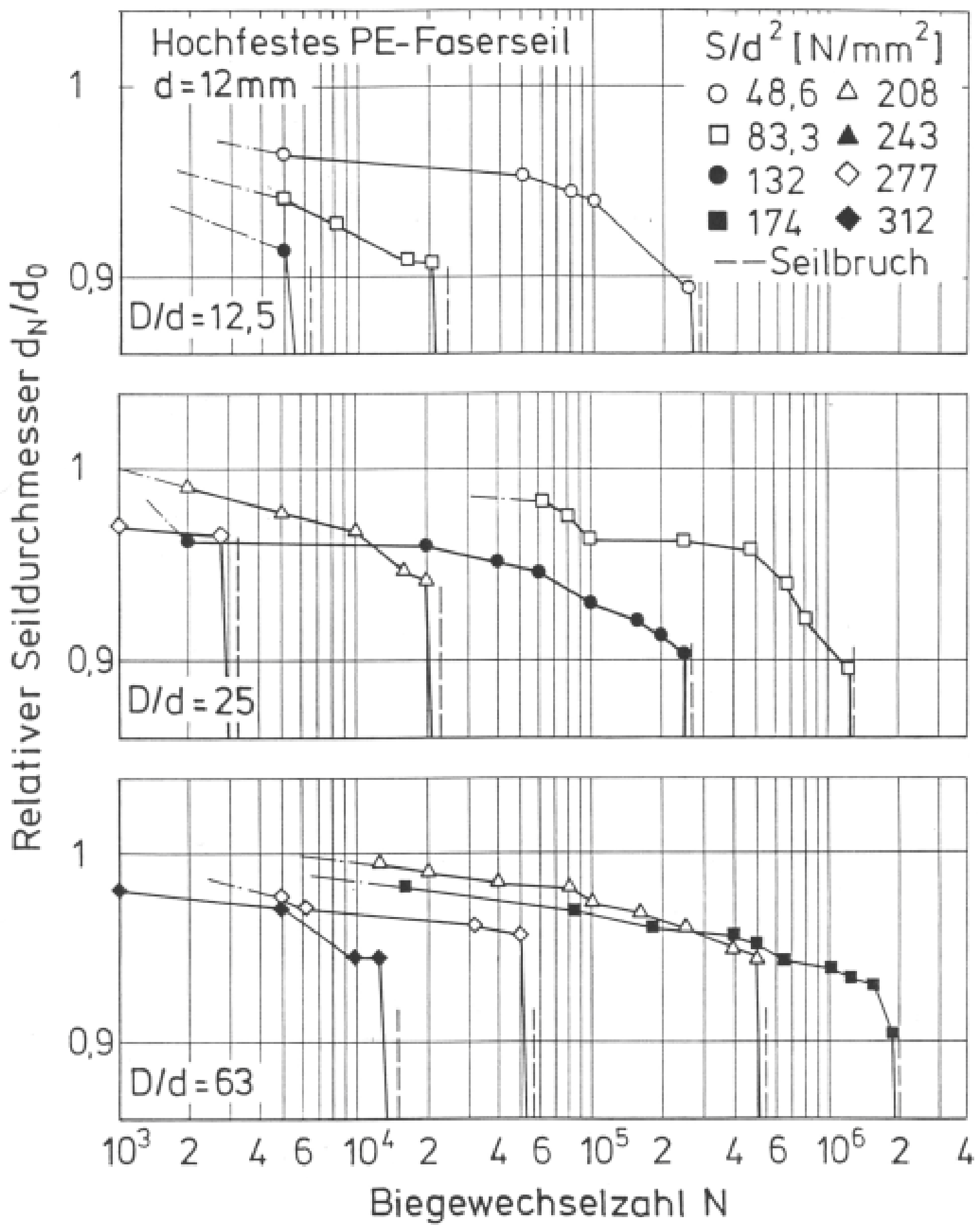

Abbildung 11: Seildurchmesser im Verlauf der Dauerbiegeversuche

Ein Schritt in die Richtung einer zuverlässigen Erkennbarkeit der Ablegereife, und zwar abgekoppelt von starren Inspektionsintervallen, ist mit den Aramidfaserseilen der Schindler AG für die Anwendung im Treibscheibenaufzug gemacht (Abb. 9). Die Erkennung der Ablegereife des Seiles, und zwar rechtzeitig bevor ein gefährlicher Zustand eintritt, ist wesentlich für den Einsatz in der sicherheitsrelevanten Anwendung Personenaufzug, aber selbstverständlich auch für andere Anwendungen der Fördertechnik, des Materialflusses und der Logistik. Bei dem Aramidfaserseil sind die tragenden Litzen aus einem Verbund aus Aramidfasern und einem Harz hergestellt und vor mechanischen Beschädigungen und dem schädigendem Einfluss von UVStrahlung durch einen Polyurethanmantel nachhaltig geschützt. Allerdings sind dadurch visuelle und taktile Inspektionen der tragenden Seillitzen nicht mehr möglich. Zur Erkennung der Ablegereife sind in die Litzen der äußeren Lage kurzdehnende, elektrisch leitende Kohlefasern eingearbeitet, deren Biegewechselfestigkeit unter 
der der tragenden Aramidfasern liegt. Die Dehnung der Kohlefasern ist auf der Basis von Dauerbiegeversuchen so eingestellt worden, dass sie früher brechen als die Aramidfasern, ihre elektrische Leitfähigkeit verlieren und somit die Ablegereife anzeigen. Die Prüfung der Seile in den Endverbindungen ist bei der gewählten Methode einschlossen. Der Vorteil der Kohlefasern liegt zusätzlich in der Möglichkeit der ständigen Überwachung (auch bei jeder Fahrt), unabhängig von starren, langen Prüfintervallen.

\section{Treibfähigkeit}

Die Treibfähigkeit ist für den sicheren Betrieb von Treibscheibenaufzügen eine wesentliche Komponente. Für den Normalbetrieb, das Beladen des Fahrkorbs und den Nothalt muss eine ausreichende Treibfähigkeit verfügbar sein. Die Treibfähigkeit des Aramidfaserseils auf Rundrillen ist am IFT auf dem sogenannten Treibfähigkeitsprüfstand untersucht worden (Abb. 12). Dabei sind die Schlupf-Seilkraftverhältniskurven für die realistischen Umgebungsbedingungen - trocken - Wassernebel - wieder getrocknet - untersucht worden. Bei den Treibfähigkeitsversuchen konnte keine zu kleine Treibfähigkeit und kein drastischer Rückgang der Treibfähigkeit durch die simulierten Umgebungsbedingungen festgestellt werden. Die Kombination Rundrille mit Aramidfaserseil in der einfachen Umschlingung stellt eine ausreichend hohe Treibfähigkeit zur Verfügung. Die Treibfähigkeit ist bei dem untersuchten Seil auch nach oben begrenzt. Durch die Begrenzung der maximalen Treibfähigkeit wird bei einer unkontrollierten Fahrbewegung nach oben oder beim ungewollten Aufsetzen des Gegengewichts in der Schachtgrube das Ziehen des Fahrkorbs unter die Schachtdecke verhindert.



Abbildung 12: Treibfähigkeitsversuche mit hochfesten, laufenden Faserseilen

\section{Zusammenfassung}

In modernen fördertechnischen Maschinen und Materialflusssystemen steigen die Anforderungen an die Tragmittel. Stahldrahtseile, die in den Seiltrieben über Scheiben laufen und/oder aufgetrommelt werden, decken dabei den größten Teil der meist sicherheitsrelevanten Anwendungen ab. Jüngere Untersuchungen des IFT zeigen, dass laufende Faserseile in speziellen Anwendungen eine Alternative zu den Stahldrahtseilen sind. Eine vollständige Substitution der Stahldrahtseile durch die Faserseile wird es aber nicht geben und ist auch nicht sinnvoll. Vielmehr muß der Anwendung entsprechend das Tragmittel zielgerichtet zugeordnet werden. Welche Chancen sich hier eröffnen, ist nicht nur in der vorne zitierten Steigerungen der Lebensdauer von Faserseilen im Vergleich zu Stahldrahtseilen im Aufzugbau zu erkennen. Beispielsweise sind im Anwendungsfall der Kranseile, 
vor allen im Automobilkranbereich mit Faserseilen bis zu 30\% Masseeinsparungen pro laufendem Meter Seil möglich. Da aber hochfeste Faserseile im Aufzugsbereich und im Kranbereich aufgrund der unterschiedlichen Anforderungen, denen sie ausgesetzt sind, völlig unterschiedliche Konstruktionen haben müssen, ist ein langer Entwicklungs- und Innovationsprozess notwendig. Dieser Entwicklungs- und Innovationsprozess soll durch diese Veröffentlichung beschleunigt werden, da nach Meinung der Autoren großes wirtschaftliches Potenzial und neuartige Alleinstellungsmerkmale gegeben sind.

\section{Literatur}

[Feyrer00]

[Vogel91]

[Vogel98]

[Vogel02]

[Vogel02]

[Vogel03]

[Wehking98]

[Wehking00]
Feyrer, Klaus: Drahtseile. Springer-Verlag Berlin 2000

Vogel, Wolfram, Feyrer, Klaus: Hochfestes Faserseil beim Lauf über Seilrollen. Draht 42 (1991) 11, S. 814-818. Englisch: WIRE 42 (1992) 5, S.455-458

Vogel, Wolfram: Dauerbiegeversuche an gedrehten und geflochtenen Faserseilen aus hochfesten Polyethylenfasern. Technische Textilien 41 (1998) 3, S. 126-128. Englisch: Bending tests with high-strength PE fiber ropes. Technical Textiles 41 (1998) 5, E 39-E40

Vogel, Wolfram: Einfluss der Schlaglänge auf die Lebensdauer laufender hochfester Faserseile. EUROSEIL 121 (2002) 3, S. 57/58

Vogel, Wolfram: Atlasseile beim Lauf über Scheiben. EUROSEIL 121 (2002) 4, S. $64 / 65$

Vogel, Wolfram: Tragmittel für Treibscheibenaufzüge. LIFT-REPORT 29 (2003) 5, S. 6-18. Englisch: Suspension means for traction elevators. LIFT-REPORT 29 (2003) 5, p. 19-28

Wehking, Karl-Heinz: Endurance of high-strength-fibre ropes running over pulleys. OIPEEC Round Table Reading 1998

Wehking, Karl-Heinz: Lebensdauer und Ablegereife von Aramidfaserseilen in Treibscheibenaufzügen der Schindler AG. Interner Forschungsbericht, Dezember 2000, Ebikon/Schweiz 